\title{
Self-Limiting Covalent Modification of Carbon Surfaces: Diazonium Chemistry with A Twist
}

\author{
Miriam C. Rodríguez González, ${ }^{a *}$ Anton Brown, ${ }^{a}$ Samuel Eyley, ${ }^{b}$ Wim Thielemans, ${ }^{b}$ Kunal S. Malia* and \\ Steven De Feyter ${ }^{*}$
}

\begin{abstract}
aDepartment of Chemistry, Division of Molecular Imaging and Photonics, KU Leuven, Celestijnenlaan 200F, B-3001 Leu-ven, Belgium, bDepartment of Chemical Engineering, Sustainable Materials Lab, KU Leuven Campus Kulak Kortrijk, Etienne Sabbelaan 53, 8500 Kortrijk, Belgium
\end{abstract}

Corresponding authors: miriamcandelaria.rodriguezgonzalez@kuleuven.be, kunal.mali@kuleuven.be, steven.defeyter@kueluven.be

\begin{abstract}
:
The chemistry of carbon surfaces has regained traction in recent years in view of its applicability towards covalent modification of a variety of (2D) materials. A general requisite is the formation of a dense and well-defined monolayer of aryl groups covalently bound to the surface. Given the use of reactive chemistries however, it is often not easy to achieve precise control over the monolayer growth while maintaining high grafting densities. Here we present a straightforward experimental protocol for the fabrication of well-defined covalent monolayers onto the surface of graphite. Using a combination of surface analytical tools, we demonstrate that the ascorbic acid mediated dediazoniation of aryldiazonium salts leads to self-limiting growth of monolayers with high grafting densities. The aryl radicals preferentially attach to the basal plane of the substrate and once the surface is covered with a covalent monolayer, the surface reaction does not proceed further to an appreciable extent. The layer thickness of the covalent films was measured using atomic force microscopy whereas the grafting efficiencies were assessed using Raman spectroscopy. The chemical composition of the grafted films was studied using X-ray photoelectron spectroscopy whereas scanning tunneling microscopy provided nanometer scale insight into the structure of the covalent films. Mechanistic aspects of the process are also discussed. The self-terminating chemistry described here is a new addition to the synthetic armory for covalent modification of materials and sets a strong foundation for achieving precise nanoscale control over the covalent functionalization process.
\end{abstract}




\section{Introduction}

Chemically modified surfaces are omnipresent in our day-to-day lives. The chemical modification often consists of an organic coating attached to the surface. While polymers are the most commonly used surface modifiers, there is an increasing interest in strongly adherent coatings where the organic film is covalently bound to the solid surface. Such robust organic coatings, which are typically composed of aryl groups, not only serve as protective layers but they also often impart specific functionality to the surface.

Aryldiazonium salts are one of the most popular surface modifiers used for the covalent modification of metal, semiconductor, polymer, and carbon-based surfaces. ${ }^{1}$ The modification process consists of the reduction of the aryldiazonium ion in the vicinity of the surface to be modified. The salt undergoes decomposition to yield a highly reactive aryl radical that attaches itself to the surface with concomitant loss of nitrogen $\left(\mathrm{N}_{2}\right)$ gas in a process commonly referred to as grafting. Although the aryldiazonium salts are known to undergo spontaneous decomposition in the presence of certain substrates $^{2}$, very often their reductive decomposition is initiated using electrochemical, ${ }^{3}$ photochemical $^{4}$ or chemical ${ }^{5,6}$ means as the latter methods provide a greater degree of control over the grafting process. Covalently modified surfaces prepared using diazonium chemistry have potential applications in diverse range of fields including sensing, ${ }^{7}$ catalysis, ${ }^{8}$ energy storage systems, ${ }^{9}$ anti-icing surfaces, 10,11 to name a few. Diazonium chemistry has recently found prominence in the covalent functionalization of 2D materials including graphene, ${ }^{12} \mathrm{MoS}_{2}{ }^{13}$ and black phosphorus. ${ }^{14,15}$

A number of aforementioned applications require well-defined, densely grafted films. Typically, covalently grafted monolayers comprising a high density of grafted aryl groups are preferred when welldefined structure is a prerequisite. Such monolayers are desirable on multiple grounds. First, they allow secondary (post-grafting) functionalization in a relatively straightforward manner where additional functionality can be readily added to the interface. Second, a well-defined monolayer can serve as a coupling platform for interfacing two different types of materials. Third, the electron transfer across monolayers is more efficient than that across multilayered, thick films. Such functionalized yet nonpassivated surfaces are highly desirable in electrocatalysis, and electrochemical sensors. ${ }^{7,8}$

Diazonium chemistry, despite its wide popularity however, does not readily yield the desired well-defined monolayers. The fast and efficient functionalization of surfaces comes at the expense of poor control over the structure of the covalent film. Given their highly reactive nature, the aryl radicals generated from the diazonium salt not only react with the surface but also attack the surface-attached aryl groups thereby leading to dendritic polyaryl growth forming multilayers. ${ }^{16}$ Such films are illdefined and exhibit poor grafting densities. In general, the tendency to form multilayers is often associated with poor density/efficiency of grafting. ${ }^{17}$

The discussion on monolayers versus multilayers figures prominently in the published literature. ${ }^{18}$ Different strategies have been employed to control the grafting process in a way that welldefined monolayers are formed while maintaining high grafting densities. ${ }^{19}$ One of the first tactic to this end was to install a cleavable link in the para position of the aryldiazonium salt. This link could be 
cleaved chemically after the grafting onto the surface to obtain monolayer films. ${ }^{20,21}$ The incorporation of bulky groups in one or more free positions on the aryldiazonium salts is also a popular strategy. Given that the polyaryl growth most often occurs via the addition of aryl radicals onto the available positions of the grafted aromatic rings, installation of sterically hindering groups such as tert-butyl units in the 3 , 5-position of the aryldiazonium salt prevents the side reaction leading to monolayer growth. ${ }^{17,22}$ Ionic liquids have been employed as reaction media to influence the grafting kinetics. It has been argued that the higher viscosity of the medium reduces the diffusion of the aryl radicals across the interface thereby providing control over the layer growth. ${ }^{23}$ Extensive work has been carried out in grafting monolayer films using radical inhibitors such as 2,2-diphenyl-1-picrylhydrazyl (DPPH) which prevent multilayer growth providing relatively homogeneous films. ${ }^{24,} 25$ Additionally, application of high overpotentials during the electrografting process has also been suggested as a general method to obtain monolayers. ${ }^{26}$

While the aforementioned strategies have evolved within the electrochemistry domain, there have been parallel attempts to achieve efficient grafting onto various surfaces using chemical activation via the use of external reducing agents. Both homogeneous and heterogeneous reductive processes have been used. Iodide-, ${ }^{6}$ and hypophosphorus acid ${ }^{27,} 28$ mediated dediazoniation and subsequent grafting occurs under homogeneous conditions where the reducing agent and the diazonium salt are in the same phase whereas iron powder-mediated reduction ${ }^{29}, 30$ involves a two-phase process. The external reducing agents have been proposed to work via two distinct mechanisms (vide infra). The so-called 'outer-sphere' mechanism involves the direct reduction of the diazonium salt by the reducing agent. The 'inner-sphere' mechanism on the other hand, involves the initial reaction of the reducing agent with the aryldiazonium salt leading to an intermediate product that subsequently decomposes to the corresponding aryl radical. Antioxidants such as ascorbic acid ${ }^{31}$ and gallic acid ${ }^{32}$ have been reported to trigger dediazoniation via the inner-sphere mechanism during the $\mathrm{C}-\mathrm{H}$ arylation of (hetero)arenes. Ascorbic acid mediated dediazoniation and grafting onto a polymer has been described earlier. ${ }^{33}$

The chemical activation of diazonium salts is an attractive approach as it is relatively simple compared to the electrochemical grafting approach. More importantly, it does not require the substrate to be conductive thus widening the scope of the grafting to non-conductive surfaces. Finally, the chemical activation strategy allows covalent modification of surface-supported substrates as well as that of material suspensions. This latter facet is particularly important given the growing interest in the application of diazonium chemistry for the covalent functionalization of 2D materials.6,12-15 In general, simple chemical protocols that yield densely grafted monolayers of aryl groups are highly sought after.

Here we report a self-terminating grafting process for the covalent functionalization of carbonbased materials using ascorbic acid mediated dediazoniation. Highly oriented pyrolytic graphite (HOPG) was used as a model substrate. We demonstrate using detailed atomic force microscopy (AFM) measurements that the ascorbic acid mediated dediazoniation of typical diazonium salts leads to preferential formation of covalent thin films where the layer thickness is limited to a monolayer. The self-limiting nature is inherent to the process and monolayers were obtained irrespective of 

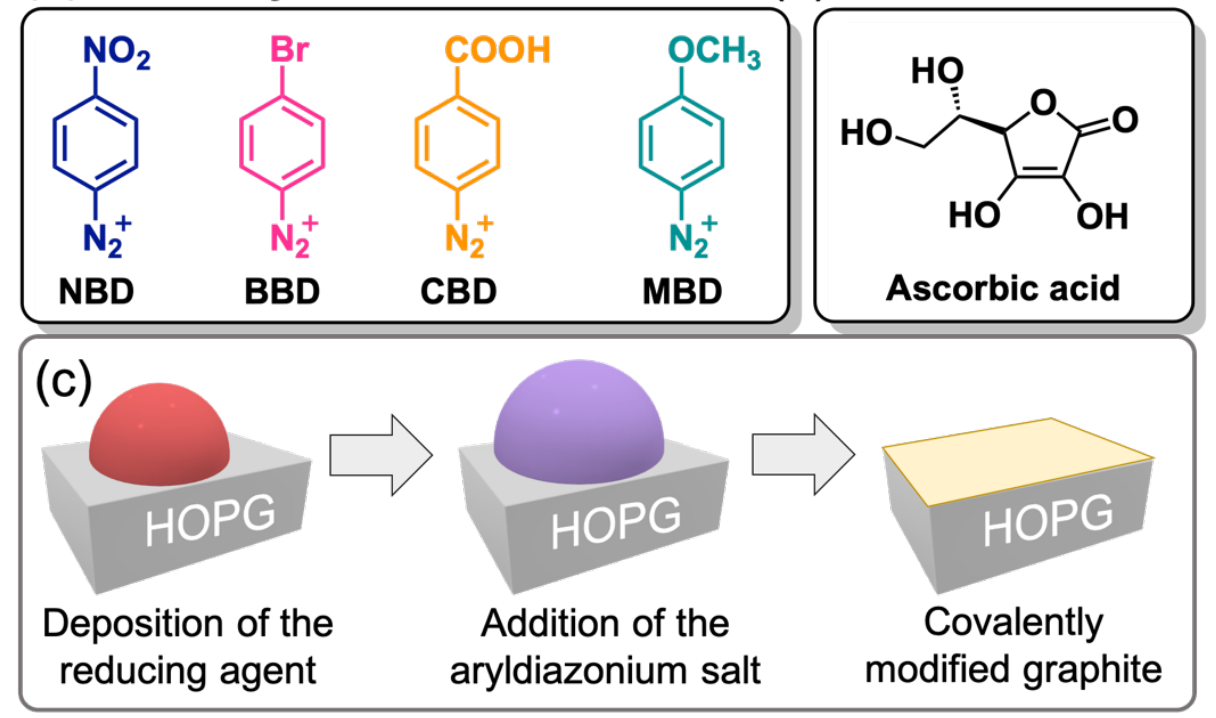

Scheme 1. Molecular structures of the (a) aryldiazonium salts and (b) ascorbic acid. (c) The covalent grafting protocol.

experimental conditions such as the concentration of the diazonium salt or the reaction time. Raman spectroscopy was employed as a primary characterization tool to quantify the efficiency of covalent grafting which revealed high grafting densities for the diazonium precursors used. X-ray photoelectron spectroscopy (XPS) was used to obtain information on the chemical composition of the grafted layers whereas scanning tunneling microscopy (STM) provided morphological information at the nanometer scale.

\section{Results and discussion:}

Scheme 1a shows the molecular structures of the diazonium salts used in this study. These precursors were chosen due to the varying degree of (de)activating effect of the functional groups in the para position. $-\mathrm{NO}_{2}$ is strongly electron withdrawing in nature whereas $-\mathrm{OCH}_{3}$ is strongly electron donating. $-\mathrm{COOH}$ and $-\mathrm{Br}$ on the other hand, are moderately and weakly electron withdrawing, respectively. The covalent grafting experiments were carried out by simply drop casting the aqueous solutions of ascorbic acid (Scheme 1b) and the diazonium salt in a sequential fashion (Scheme 1c) onto a freshly cleaved HOPG. The reaction was stopped after 10 minutes by thoroughly rinsing the samples using acetonitrile and water followed by drying under a stream of argon. The dried samples were subjected to a thorough spectroscopic and microscopic characterization as described below.

The chemical composition of the covalently grafted layers was studied in detail using XPS. The survey spectra presented in Figure S1 in the ESI were used to determine the overall elemental composition of the four samples. Figure 1a shows the N 1s spectrum for the NBD modified graphite sample. The peak at $405.9 \mathrm{eV}$ is indicative of the presence of the expected $-\mathrm{NO}_{2}$ groups. An additional peak at $400.1 \mathrm{eV}$ could arise due to the presence of reduced nitrogen species such as $-\mathrm{N}=\mathrm{N}-$ 
(a)

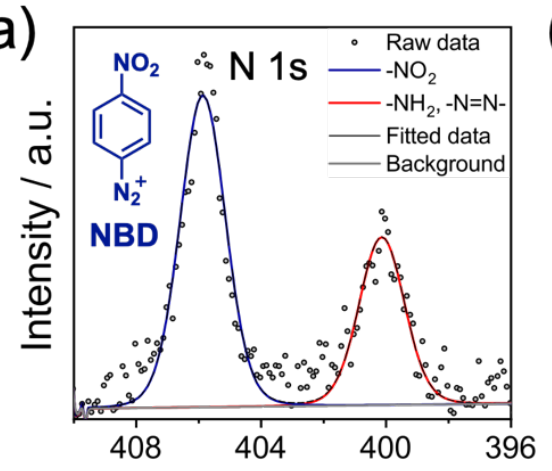

Binding energy / eV

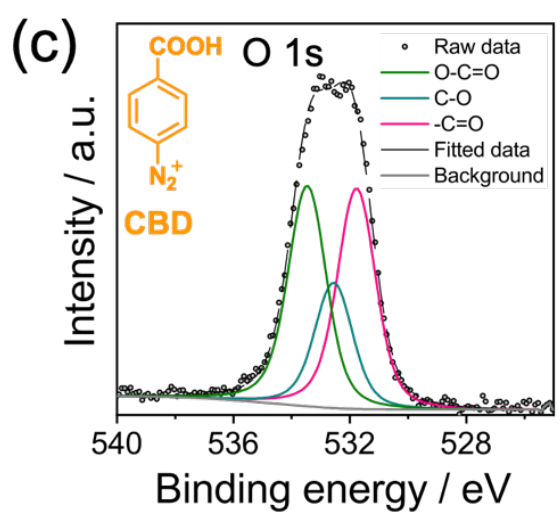

(b)

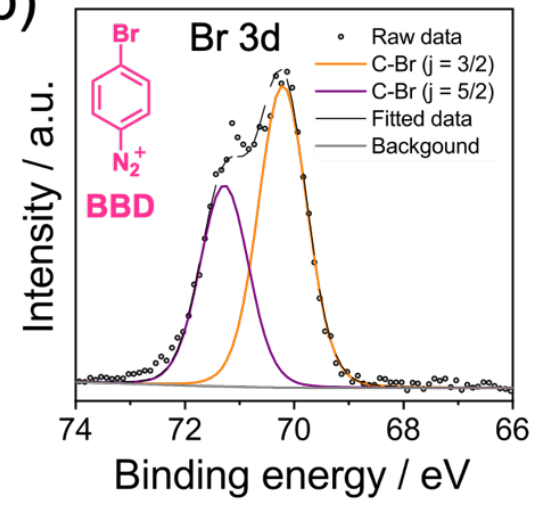

(d)

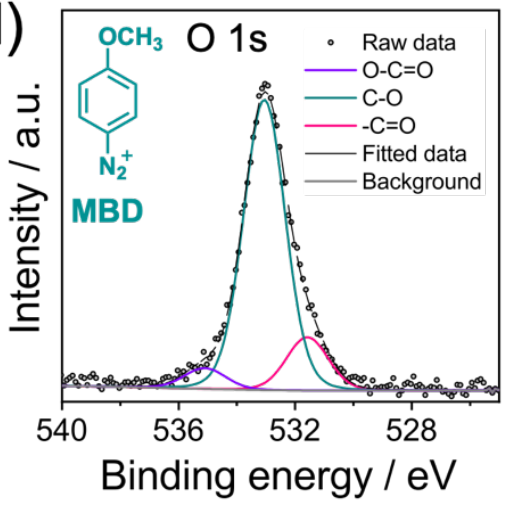

Figure 1. High-resolution XPS analysis of the covalently functionalized graphite samples.

or $-\mathrm{NH}_{2}$ groups. ${ }^{34}$ The appearance of these peaks has been linked to the partial reaction of the diazonium cations with the surface grafted units, ${ }^{35}$ and the photochemical reduction of the $-\mathrm{NO}_{2}$ groups by X-ray irradiation, ${ }^{36}$ respectively. The bromine $3 \mathrm{~d}$ region for the $\mathbf{B B D}$ modified sample is shown in Figure $1 \mathrm{~b}$. The analysis of the bromine $3 \mathrm{~d}$ peak shows a $3 \mathrm{~d}_{5 / 2}$ binding energy consistent with $\mathrm{C}-\mathrm{Br}$ bond at $70.2 \mathrm{eV}$ together with the corresponding $3 \mathrm{~d}_{3 / 2}$ contribution. ${ }^{34}$ The interpretation of the signals in the carbon and the oxygen region for the other two systems was not straightforward due of the plausible presence of residual ascorbic acid in the sample, despite thorough rinsing. Notwithstanding the overlap of the signature peaks of the functional groups attached to the aryl units with those of residual ascorbic acid, some important differences can be seen when the 0 1s spectra for CBD and MBD are compared. For CBD, the 01 s spectrum (Figure 1c) consists of three environments. The peaks at $531.8 \mathrm{eV}$ and $533.5 \mathrm{eV}$ are due to the carboxyl moiety $(\mathrm{O}=\mathrm{C}$ and $\mathrm{O}-\mathrm{CO}$ respectively), while the smaller peak at $532.5 \mathrm{eV}$ is due to $\mathrm{O}-\mathrm{C}$ from residual contamination. For MBD, the expected methoxy group is also confirmed in the $\mathrm{O}$ 1 s spectrum by the component at $533.1 \mathrm{eV}$ (Figure 1d), while the contaminating $\mathrm{O}=\mathrm{C}$ species is also present at $531.6 \mathrm{eV}$. The C1s component for the samples is included in Figure S2 in the ESI. While the XPS spectra provide detailed information about the chemical composition of the organic layers, they do not necessarily prove their covalent grafting onto the surface. Hence, the primary evidence for covalent attachment of the aryl radicals to the graphite surface was obtained using Raman spectroscopy. ${ }^{37}$ 

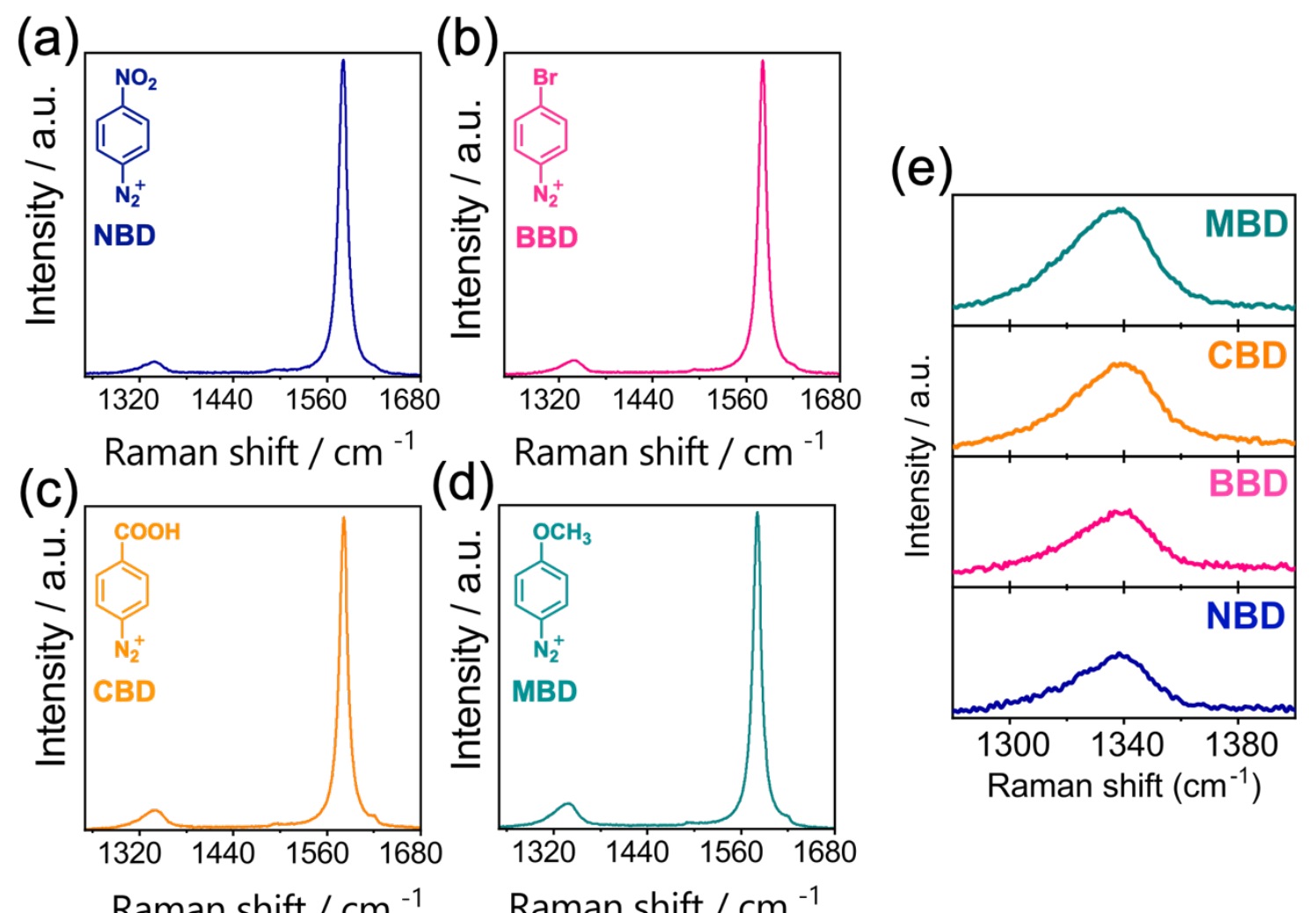

Figure 2. (a-d) Raman spectra of the covalently grafted films. (e) The same spectra on the smaller scale highlighting the D-band, the normalized intensity of which increases in the order: $\mathbf{N B D}<\mathbf{B B D}<\mathbf{C B D}<$ MBD. $\left(\mathrm{C}_{\text {diazonium salt }}=\mathrm{C}_{\text {ascorbic acid }}=10 \mathrm{mM}\right.$, reaction time $=10$ minutes $)$

Figure 2 shows representative Raman spectra of graphite substrates functionalized using the different diazonium salts. All the Raman spectra show a well-discernible D-band $\left(\sim 1340 \mathrm{~cm}^{-1}\right)$, which is indicative of the formation of $\mathrm{sp}^{3}$ defects in the graphite due the covalent attachment of the aryl groups. The ratio of the intensity of the D- and the G-band $\left(\sim 1580 \mathrm{~cm}^{-1}\right)[\mathrm{I}(\mathrm{D}) / \mathrm{I}(\mathrm{G})]$ was used to quantify the degree of grafting. Note that the relative intensities of the D- and G-bands in the case of graphite and graphene are different. The $\mathrm{G}$ band in the Raman spectrum of graphite is obviously more intense than that in graphene. Hence it readily follows that the $\mathrm{I}(\mathrm{D}) / \mathrm{I}(\mathrm{G})$ ratios for functionalized HOPG are lower compared to that for functionalized single layered graphene.6,17

The I(D)/I(G) ratio obtained here for NBD is comparable to that reported by us recently using electrochemical grafting for the same NBD concentration. ${ }^{38}$ Table 1 provides the I(D)/I(G) ratios for the different systems studied here. These values indicate that the grafting efficiency of NBD which carries the electron withdrawing $-\mathrm{NO}_{2}$ group is the lowest whereas that of $\mathbf{M B D}$ carrying the electron donating $-\mathrm{OCH}_{3}$ group is the highest. The I(D)/I(G) ratios for BBD and CBD are in between these two extremes. The trend observed here is in line with that obtained upon electrografting of aryl groups carrying activating and deactivating functional groups. ${ }^{39}$ We have recently argued that amongst other factors, the relative position of the singly occupied molecular orbital (SOMO) energy levels of the aryl radicals with respect to the graphite Fermi level determines their reactivity and thus subsequently the efficiency of grafting. 40 

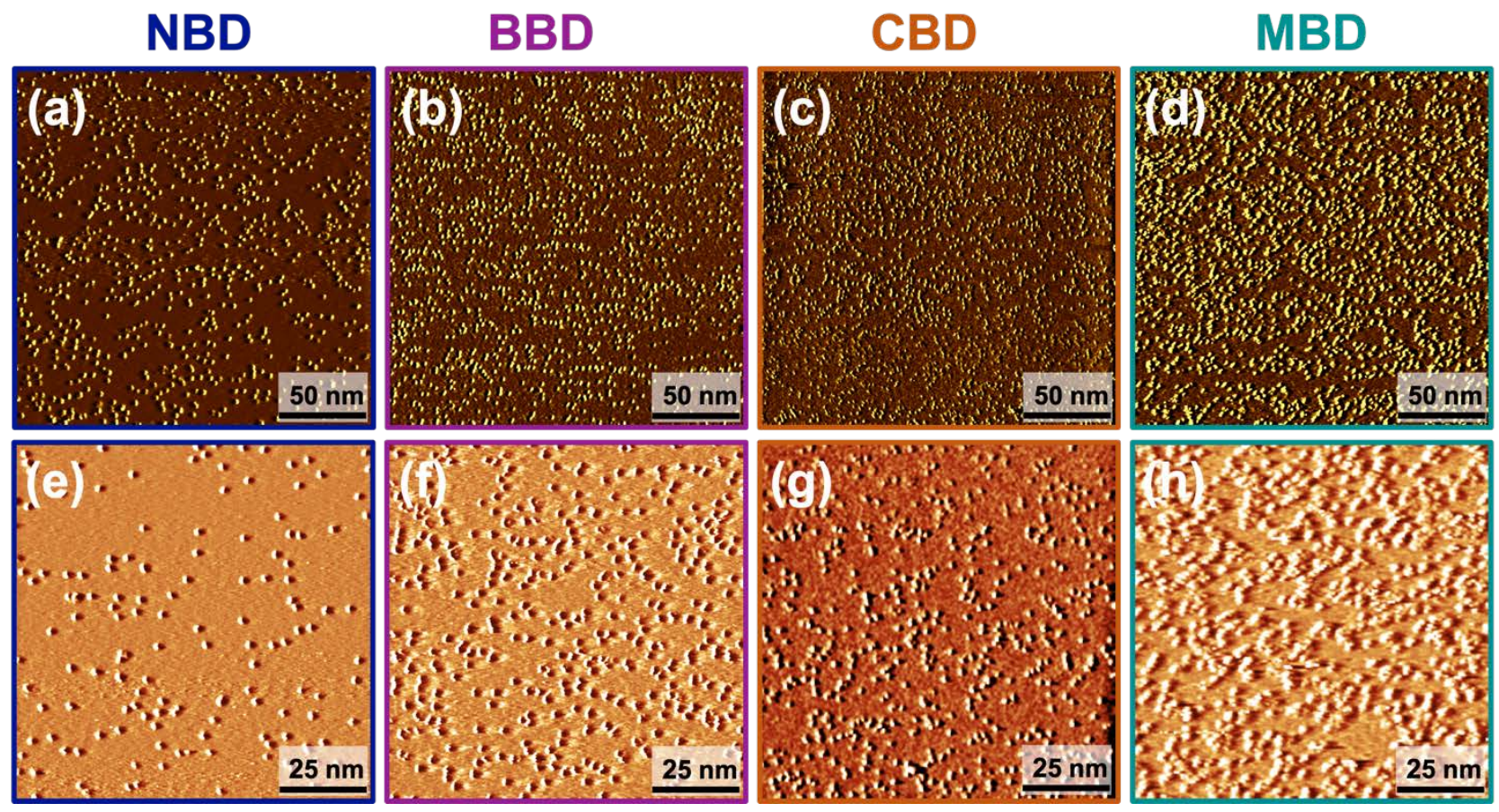

Figure 3. (a-d) Representative large scale STM images of the covalently modified graphite substrates. (e-h) Representative small scale STM images of the covalently modified graphite substrates. $\left(\mathrm{C}_{\text {diazonium }}\right.$ salt $=C_{\text {ascorbic acid }}=10 \mathrm{mM}$, reaction time $=10$ minutes) Imaging parameters: $I_{t}=0.08 \mathrm{nA}, V_{\text {bias }}=-700 \mathrm{mV}$ )

Having confirmed high grafting efficiencies using Raman spectroscopy, we further characterized the grafted surfaces at the nanometer scale using scanning probe microscopy to develop a molecular level understanding of the grafting process. To this end, the modified substrates were first probed using STM. We have employed STM to image grafted aryl groups on the surface of graphite and single layer graphene in the recent past. $17,41,42$ STM measurements provide nanoscale insight in to the efficiency of the grafting process. Figure 3 shows representative large- and small scale STM images of covalently modified graphite using the different precursors. The images show that the surface is covered with spherical bright features which are attributed to the covalently bound aryl groups. The trend in the grafting densities obtained from the Raman measurements is confirmed by the STM data wherein the large-scale images clearly show an increase in the number of bright features in the following order: NBD $<$ BBD $<$ CBD $<$ MBD. We note, however, that the STM data is somewhat qualitative as the STM tip tends to cause unintentional removal of the grafted aryl units upon repetitive scanning. ${ }^{38}$ Although STM provides molecular resolution data, it does not offer reliable height information which is needed to obtain layer thickness. 
Table 1. I(D)/I(G) ratio, layer thickness, and roughness values for the covalently modified graphite substrates prepared using the different diazonium salts. The roughness values were obtained from 0.6 $\mu \mathrm{m} \times 0.6 \mu \mathrm{m}$ AFM images.

\begin{tabular}{cccc}
\hline Precursor & I(D)/I(G) & Thickness (nm) & Roughness (nm) \\
\hline NBD & $0.033 \pm 0.002$ & $0.9 \pm 0.1$ & $0.37 \pm 0.01$ \\
\hline BBD & $0.042 \pm 0.002$ & $1.1 \pm 0.2$ & $0.31 \pm 0.01$ \\
\hline CBD & $0.063 \pm 0.002$ & $1.0 \pm 0.1$ & $0.20 \pm 0.01$ \\
\hline MBD & $0.074 \pm 0.003$ & $0.8 \pm 0.1$ & $0.33 \pm 0.01$ \\
\hline
\end{tabular}

AFM measurements were carried out for obtaining the layer thickness of the modified substrates and to evaluate the general morphology of the covalent films. Figure 4 shows representative AFM images of grafted substrates obtained using the different diazonium precursors. In all cases, a homogeneously distributed film with a granular structure and a surface coverage between 80 and $90 \%$ was observed. This granular structure has been reported previously for aryl-based layers on HOPG surfaces. ${ }^{34,} 43$ The covalent films obtained from NBD show topographically lower areas with depths smaller than $1 \mathrm{~nm}$. This information however, is not enough to conclude on the layer thickness. In order to obtain reliable information, small parts of the films were locally scratched away using the AFM tip in the contact mode. The layer thickness was then measured across such scratched areas. The thickness was found to be between $0.8 \mathrm{~nm}$ and $1.0 \mathrm{~nm}$ in all four cases (see Table 1). Figure 4e shows a representative AFM image of the surface after performing such local scratching experiment which clearly reveals a layer thickness of $0.9 \mathrm{~nm}$ (also see Figure S3 in the ESI). Given that the height of an aryl group orthogonally bound to the surface through a C-C bond is $\sim 0.8 \mathrm{~nm}$, the AFM data confirms that the films formed in all the cases have a limiting monolayer thickness. The covalent monolayers are quite smooth as indicated by the RMS roughness values presented in Table 1. Furthermore, the roughness values do not change significantly upon changing the scan size confirming their structural homogeneity across the surface (see Figure S4 in the ESI).

The self-limiting nature appears to be inherent to the process and does not depend on the experimental conditions such as the concentration of the diazonium salt, reaction time or the type of the diazonium precursor (Figure S5 in the ESI). The charge-transfer properties of the functionalized surfaces were examined using the redox response of ferrocene across the covalent film. (Figure S6 in the ESI). A good reversible response from this redox probe across the covalent films obtained from all the four diazonium precursors confirms that the functionalized surfaces have excellent charge-transfer properties. These experiments further confirm that the surface modification protocol can be applied to different materials maintaining their good charge-transfer properties. 

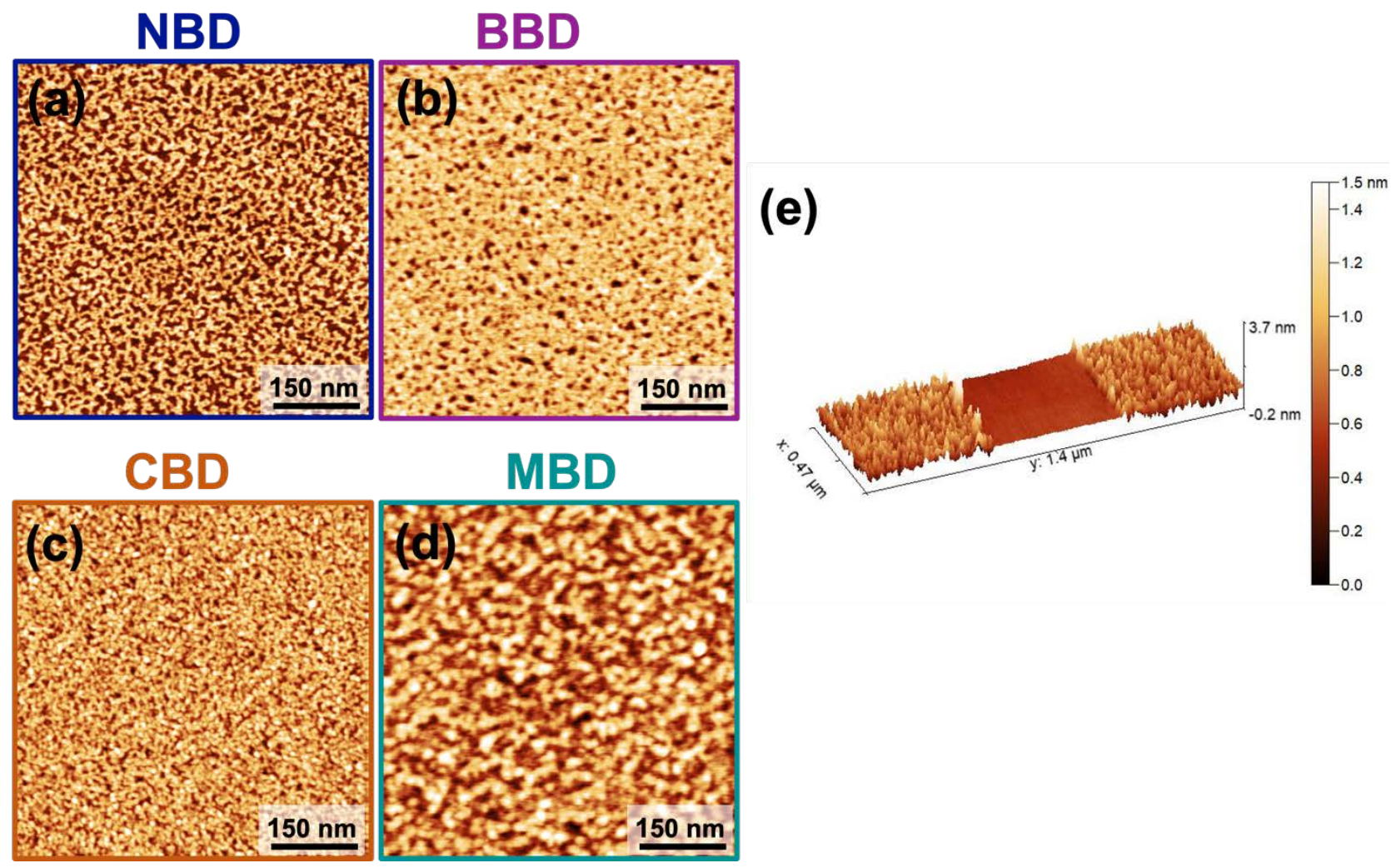

Figure 4. (a-d) Representative AFM height images of the covalently modified graphite substrates. (e) 3D rendering of a small area scratched away using AFM tip for the estimation of layer thickness. $\left(C_{\text {diazonium salt }}=\mathrm{C}_{\text {ascorbic acid }}=10 \mathrm{mM}\right.$, reaction time $=10$ minutes $)$. For additional AFM images of the scratching experiment, see Figure S3 in the ESI.

The exclusive formation of monolayers in all the four cases investigated here is an exciting observation given the challenges involved in obtaining well-structured dense monolayers of covalently grafted aryl groups. ${ }^{19}$ What is particularly interesting about this observation is that the multilayer growth is suppressed despite the availability of the reactive 3, 5 positions on the grafted aryl groups. Generally, for structurally similar diazonium precursors, redox inhibitors such as DPPH are needed to avoid polyaryl growth. ${ }^{24}$ Furthermore, the approach is known to work efficiently only in the case of aryldiazonium salts carrying strongly electron withdrawing groups such as $-\mathrm{NO}_{2}$. The selective formation of monolayers by direct mixing of the reagents on the surface without the need of any additional measures to control the thickness is a promising development and needs further mechanistic scrutiny. While the detailed exploration of the mechanism of the grafting process is not within the scope of this work, we discuss below the possible reaction pathways that may be responsible for the observed self-terminating nature.

As described earlier, the chemical reduction of the diazonium salts proceeds via either the 'outersphere' or the 'inner-sphere' mechanism. The former involves the direct electron transfer from the reducing agent to the diazonium salt leading to the formation of the corresponding aryl radical with the 
(a) Outer-sphere mechanism

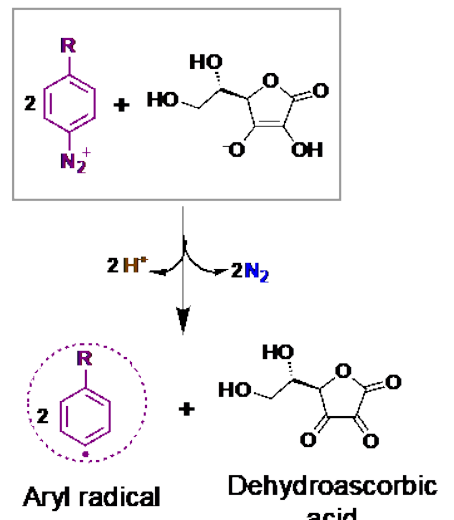

acid

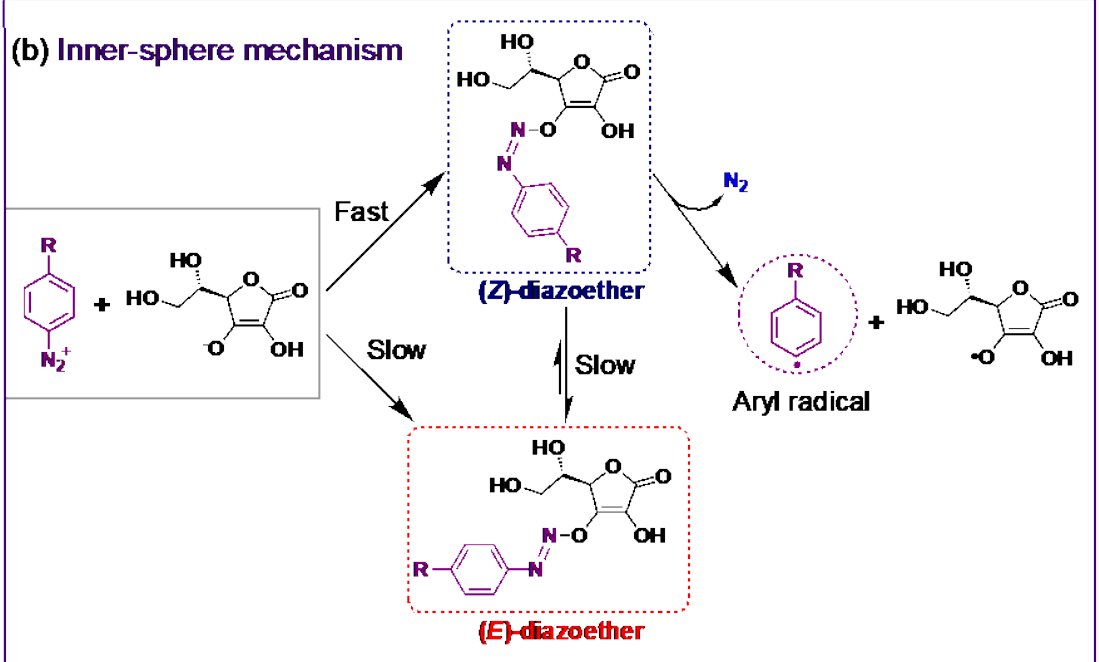

Scheme 2. Reaction pathways for the ascorbic acid mediated dediazoniation of aryldiazonium salts.

loss of $\mathrm{N}_{2}$. This redox process also yields dehydroascorbic acid as the by-product (Scheme 2a). The latter, on the other hand, proceeds via the reaction of the ascorbate ion with the aryldiazonium salt yielding an adduct via a non-redox process. These adducts have been isolated, chemically characterized and identified as the diazoethers. ${ }^{44}$ The reaction of aryldiazonium salts with the ascorbate ions occurs in three steps as depicted in Scheme 2b: (1) the rapid formation of the (Z)-diazoether; (2) partial decomposition of the $(Z)$-diazoether to yield the reduction products, and (3) the slow conversion of the $(Z)$-diazoether to the thermodynamically more stable $(E)$-diazoether. Some studies have confirmed that the $(Z)$-diazoether is formed almost exclusively, and a part of it is transformed to the $(E)$-isomer by an ionization-recombination mechanism. ${ }^{45}$ Diazoethers have been employed as a source of aryl radicals for the covalent functionalization of carbon nanotubes. It has been demonstrated that the $(E)$-isomer is virtually unreactive and that the covalent grafting on to the carbon nanotubes proceeds exclusively via the decomposition of the (Z)-diazoether. ${ }^{46}$

The self-terminating surface grafting observed here strongly indicates that the production of the aryl radicals occurs only in the presence, and the vicinity of the bare graphite surface. Once it becomes covered with a monolayer of aryl groups, the reaction is terminated. This was independently verified by carrying out the grafting reaction on a partially grafted surface. Raman and AFM measurements showed that the additional grafting occurs only in the exposed areas (indicated by increase in the I(D)/I(G) ratio) wherein the previously uncovered regions are 'filled up' by the aryl groups (as confirmed by AFM measurements) added in the second step (Figure S7 in the Supporting Information).

Based on these observations, it is tempting to conclude that the self-limiting grafting process proceeds via the surface-assisted decomposition of the $(Z)$-diazoether. The outer-sphere mechanism which directly produces the aryl radicals upon mixing of the two reagents does not appear to describe well the monolayer control observed here. Furthermore, attempts to confirm the presence of dehydroascorbic acid in the reaction mixture using GC-MS were not fruitful, possibly in view of its low 
concentration in solution. Nevertheless, it has been argued that both the reaction pathways are possible and their relative contribution depends on the exact experimental conditions. ${ }^{33}$

In the present case, when the experiment was carried out using premixed solutions instead of sequential deposition of reagents, ill-defined films with poor grafting densities were obtained (see Figure S8-S10 in the ESI). These results can be understood by considering a greater contribution from the outer-sphere mechanism where a large proportion of the aryl radicals produced via the direct reduction of the diazonium salt are consumed via dimerization or other side reactions prior to drop casting onto the surface. We propose that the two mechanisms are not mutually exclusive and it is plausible that in the presence of the graphite surface, the inner sphere mechanism dominates. The graphite surface is inherently reductive in nature and given that the decomposition of the diazoether is also a reductive process, it is not unreasonable to assume that the self-limiting covalent grafting progresses via the surface mediated decomposition of the $(Z)$-diazoether. Last but not the least, preliminary experiments carried on $\mathrm{Au}(111) /$ mica substrates using the current functionalization protocols indicate that the thickness of the grafted layers is around $1 \mathrm{~nm}$ (see AFM data in Figure S11). This observation suggests that the chemistry may remain self-limiting on substrates other than HOPG.

\section{Conclusions and outlook:}

To conclude, we have demonstrated a simple yet efficient functionalization protocol for the covalent modification of graphite. Thanks to the self-limiting nature of the reaction, high density monolayers of aryl groups were obtained preferentially irrespective of the experimental conditions and the type of the diazonium salt. The fact that the reactive chemistry does not proceed further after the formation of the first monolayer strongly implies the surface-assisted nature of the process. Selfterminating chemistries such as the one described here have enormous yet largely untapped synthetic potential. While it is demonstrated in the case of a relatively unreactive substrate here, the generality and the versatility of the process in surface modification of other materials needs to be explored further. As alluded to in the discussion, the origin of the self-limiting nature of the process remains an open question and needs an independent analytical investigation. Some of these aspects are the subject of an ongoing study.

\section{Conflicts of interest}

There are no conflicts to declare.

\section{Acknowledgements}

The authors gratefully acknowledge financial support from the Fund of Scientific Research Flanders (FWO), KU Leuven - Internal Funds. This work was in part supported by FWO under EOS 30489208. MCRG thanks the PDM fellowship from KU Leuven. AB thanks FWO for personal SB fellowship. WT further acknowledges the Provincie West-Vlaanderen for his Chair in Advanced Materials. WT and SE 
thank Research Foundation Flanders (grant G0A1219N), KU Leuven (grant C14/18/061), and the European Union's European Fund for Regional Development, Flanders Innovation \& Entrepreneurship and the Province of West-Flanders (Accelerate3 project, Interreg Vlaanderen-Nederland program) for financial support.

\section{Notes and references}

1. S. Mahouche-Chergui, S. Gam-Derouich, C. Mangeney and M. M. Chehimi, Chem. Soc. Rev., 2011, 40, 4143-4166.

2. F. Barrière and A. J. Downard, J. Solid State Electr., 2008, 12, 1231-1244.

3. D. Bélanger and J. Pinson, Chem. Soc. Rev., 2011, 40, 3995-4048.

4. M. Busson, A. Berisha, C. Combellas, F. Kanoufi and J. Pinson, Chem. Commun., 2011, 47, 1263112633.

5. M. Toupin and D. Bélanger, Langmuir, 2008, 24, 1910-1917.

6. Y. Xia, C. Martin, J. Seibel, S. Eyley, W. Thielemans, M. van der Auweraer, K. S. Mali and S. De Feyter, Nanoscale, 2020, DOI: 10.1039/D0NR03309J.

7. D. Hetemi, V. Noël and J. Pinson, Biosensors, 2020, 10, 4.

8. Q. Lenne, Y. R. Leroux and C. Lagrost, ChemElectroChem, 2020, 7, 2345-2363.

9. B. D. Assresahegn, T. Brousse and D. Bélanger, Carbon, 2015, 92, 362-381.

10. S. Gam-Derouich, J. Pinson, P. Decorse, Y. Luo, R. Herbaut, L. Royon and C. Mangeney, Chem. Commun., 2018, 54, 8983-8986.

11. H. Idriss, O. Guselnikova, P. Postnikov, Z. Kolska, P. Haušild, J. Čech, O. Lyutakov and V. Švorčík, ACS Applied Polymer Materials, 2020, 2, 977-986.

12. J. M. Englert, C. Dotzer, G. Yang, M. Schmid, C. Papp, J. M. Gottfried, H.-P. Steinrück, E. Spiecker, F. Hauke and A. Hirsch, Nature Chem., 2011, 3, 279-286.

13. K. C. Knirsch, N. C. Berner, H. C. Nerl, C. S. Cucinotta, Z. Gholamvand, N. McEvoy, Z. Wang, I. Abramovic, P. Vecera, M. Halik, S. Sanvito, G. S. Duesberg, V. Nicolosi, F. Hauke, A. Hirsch, J. N. Coleman and C. Backes, ACS Nano, 2015, 9, 6018-6030.

14. A. Hirsch and F. Hauke, Angew. Chem. Int. Ed., 2018, 57, 4338-4354.

15. C. R. Ryder, J. D. Wood, S. A. Wells, Y. Yang, D. Jariwala, T. J. Marks, G. C. Schatz and M. C. Hersam, Nature Chem., 2016, 8, 597-602.

16. P. A. Brooksby and A. J. Downard, Langmuir, 2004, 20, 5038-5045.

17. J. Greenwood, T. H. Phan, Y. Fujita, Z. Li, O. Ivasenko, W. Vanderlinden, H. Van Gorp, W. Frederickx, G. Lu, K. Tahara, Y. Tobe, H. Uji-i, S. F. L. Mertens and S. De Feyter, ACS Nano, 2015, 9, 5520-5535.

18. R. Quirós-Ovies, M. Vázquez Sulleiro, M. Vera-Hidalgo, J. Prieto, I. J. Gómez, V. Sebastián, J. Santamaría and E. M. Pérez, Chem. Eur. J., 2020, 26, 6629-6634. 
19. T. Breton and A. J. Downard, Aust J. Chem., 2017, 70, 960-972.

20. L. T. Nielsen, K. H. Vase, M. Dong, F. Besenbacher, S. U. Pedersen and K. Daasbjerg, J. Am. Chem. Soc., 2007, 129, 1888-1889.

21. K. Malmos, M. Dong, S. Pillai, P. Kingshott, F. Besenbacher, S. U. Pedersen and K. Daasbjerg, J. Am. Chem. Soc., 2009, 131, 4928-4936.

22. C. Combellas, F. Kanoufi, J. Pinson and F. I. Podvorica, J. Am. Chem. Soc., 2008, 130, 8576-8577.

23. O. Fontaine, J. Ghilane, P. Martin, J.-C. Lacroix and H. Randriamahazaka, Langmuir, 2010, 26, 18542-18549.

24. T. Menanteau, E. Levillain and T. Breton, Chem. Mater., 2013, 25, 2905-2909.

25. I. López, M. Cesbron, E. Levillain and T. Breton, ChemElectroChem, 2018, 5, 1197-1202.

26. L. Pichereau, I. López, M. Cesbron, S. Dabos-Seignon, C. Gautier and T. Breton, Chem. Commun., $2019,55,455-457$.

27. M. Pandurangappa and T. Ramakrishnappa, J. Solid State Electr., 2008, 12, 1411-1419.

28. P. Abiman, G. G. Wildgoose and R. G. Compton, Journal of Physical Organic Chemistry, 2008, 21, 433-439.

29. X. T. Le, P. Viel, P. Jégou, A. Garcia, T. Berthelot, T. H. Bui and S. Palacin, J. Mater. Chem., 2010, 20, 3750-3757.

30. V. Mévellec, S. Roussel, L. Tessier, J. Chancolon, M. Mayne-L'Hermite, G. Deniau, P. Viel and S. Palacin, Chem. Mater., 2007, 19, 6323-6330.

31. F. P. Crisóstomo, T. Martín and R. Carrillo, Angew. Chem. Int. Ed., 2014, 53, 2181-2185.

32. M. D. Perretti, D. M. Monzón, F. P. Crisóstomo, V. S. Martín and R. Carrillo, Chem. Commun., 2016, 52, 9036-9039.

33. A. Mesnage, S. Esnouf, P. Jégou, G. Deniau and S. Palacin, Chem. Mater., 2010, 22, 6229-6239.

34. M. C. R. González, A. G. Orive, R. C. Salvarezza and A. H. Creus, Phys. Chem. Chem. Phys., 2016, 18, 1953-1960.

35. P. Doppelt, G. Hallais, J. Pinson, F. Podvorica and S. Verneyre, Chem. Mater., 2007, 19, 4570-4575.

36. K. Roodenko, M. Gensch, J. Rappich, K. Hinrichs, N. Esser and R. Hunger, J. Phys. Chem. B, 2007, 111, 7541-7549.

37. A. C. Ferrari and D. M. Basko, Nat. Nanotechnol., 2013, 8, 235-246.

38. R. Steeno, M. C. Rodríguez González, S. Eyley, W. Thielemans, K. S. Mali and S. De Feyter, Chem. Mater., 2020, DOI: 10.1021/acs.chemmater.0c01393.

39. T. Menanteau, M. Dias, E. Levillain, A. J. Downard and T. Breton, J. Phys. Chem. C, 2016, 120, 44234429.

40. K. Tahara, Y. Kubo, B. Lindner, S. Hashimoto, S. Hirose, A. Brown, B. Hirsch, L. Daukiya, S. De Feyter and Y. Tobe, Langmuir, 2019, 35, 2089-2098.

41. K. Tahara, Y. Kubo, S. Hashimoto, T. Ishikawa, H. Kaneko, A. Brown, B. E. Hirsch, S. D. Feyter and Y. Tobe, J. Am. Chem. Soc., 2020, 142, 7699-7708. 
42. K. Tahara, T. Ishikawa, B. E. Hirsch, Y. Kubo, A. Brown, S. Eyley, L. Daukiya, W. Thielemans, Z. Li, P. Walke, S. Hirose, S. Hashimoto, S. De Feyter and Y. Tobe, ACS Nano, 2018, 12, 11520-11528.

43. H. Ma, L. Lee, P. A. Brooksby, S. A. Brown, S. J. Fraser, K. C. Gordon, Y. R. Leroux, P. Hapiot and A. J. Downard, J. Phys. Chem. C, 2014, 118, 5820-5826.

44. A. Dudzik, K. Jaszczuk, S. Losada-Barreiro and C. Bravo-Díaz, New J. Chem., 2017, 41, 2534-2542.

45. T. J. Broxton and D. L. Roper, The Journal of Organic Chemistry, 1976, 41, 2157-2162.

46. L. R. Powell, M. Kim and Y. Wang, J. Am. Chem. Soc., 2017, 139, 12533-12540.

\section{TOC graphic:}

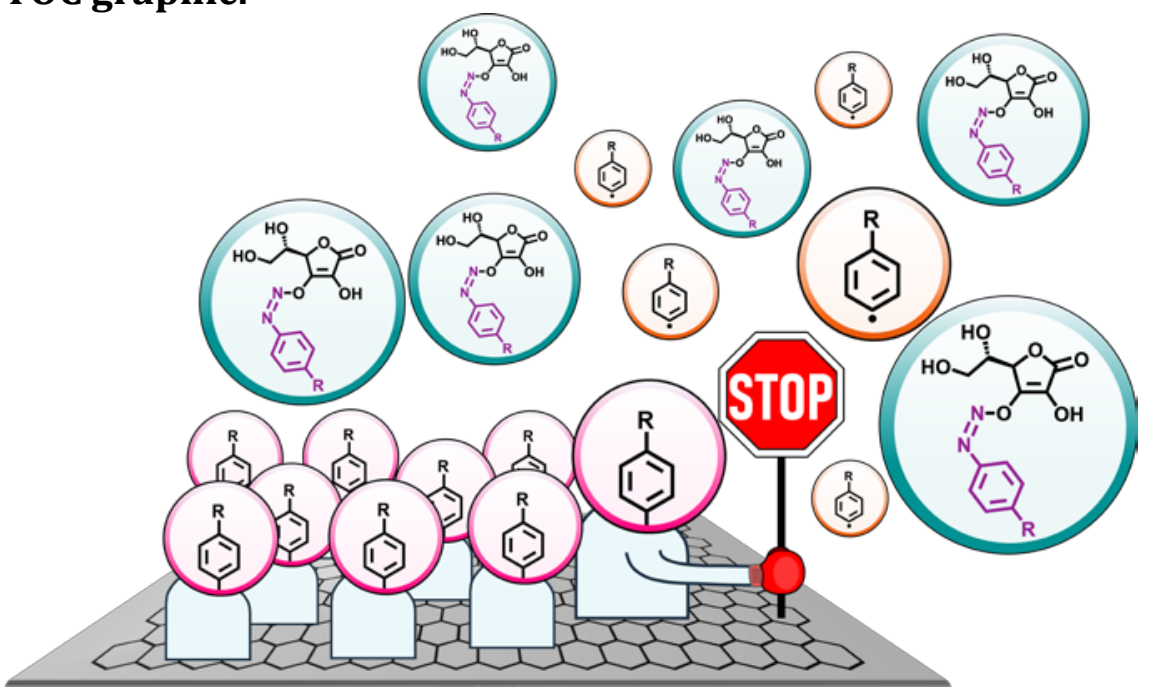

